\title{
Mass Preservation for Respiratory Motion Registration in both PET and CT
}

\author{
Elise C. Emond, Student Member, IEEE, Alexandre Bousse, Ludovica Brusaferri, Student Member, IEEE, \\ Ashley M. Groves, Brian F. Hutton, Senior Member, IEEE and Kris Thielemans, Senior Member, IEEE
}

\begin{abstract}
Registration of medical images corresponding to different respiratory states is complicated, as respiration not only moves the lungs and nearby organs, but it also causes localised density and radiotracer activity concentration changes of up to $20 \%$ in the lung. Nevertheless, few registration methods incorporate mass-preserving constraints, which could lead to suboptimal estimation of the deformation. This is especially important in the case of diffuse lung diseases for which the deformation field could provide biomarkers for pulmonary mechanical properties. This preliminary work is aimed at evaluating the impact of lung expansion in CT and PET image registration. We use patient data for which gated CT and PET acquisition data are available. We compare results of mass-preserving registration with two novel priors on the Jacobian determinant of the deformation field. Best results were observed for edge-preserving regularisation, where the mean errors in the lungs are decreased $(-17.0 \%$ for CT and $-4.8 \%$ for regularised PET using average regularisation), compared to warped images with no mass preservation.
\end{abstract}

\section{INTRODUCTION}

D ENSITY and activity variations due to compression and dilation of the lungs [1], [2] in Positron Emission Tomography (PET) reconstruction have mostly been neglected throughout the years. Motion compensation is an active area of research, usually relying on registration either on gated reconstructed images or within joint reconstruction of motion and activity [3]. Although standard registration can diminish mispositioning issues, changes in density and activity concentration should be included within the registration for accurate deformation field estimation. This could provide in return information on lung rigidity and elasticity, thus be a biomarker for lung diseases. One way to do so is to add the Jacobian determinant within the similarity measure to approximate the volume changes, linked to density and activity changes [4]-[6]. Mass-preserving registration is however nontrivial and therefore regularisation and similarity measures need to be optimised especially in the presence of noisy data.

Manuscript received December 13, 2019. E. C. Emond was supported by GlaxoSmithKline (BIDS3000030921). A. Bousse was funded by GE Healthcare. L. Brusaferri was supported by Siemens Healthineers and the UCL Impact Scheme, the EPSRC-funded UCL Centre for Doctoral Training in Medical Imaging (EP/L016478/1) and the UCL Institute of Healthcare Engineering. Research was supported by NIHR UCLH Biomedical Research Centre.

E. C. Emond is with the Institute of Nuclear Medicine, University College London, London NW12BU, UK (e-mail: elise.emond.16@ucl.ac.uk).

L. Brusaferri, K. Erlandsson, A. M. Groves, B. F. Hutton and K. Thielemans are with the Institute of Nuclear Medicine, University College London, London NW1 2BU, UK.

A. Bousse was with the Institute of Nuclear Medicine, University College London, London NW1 2BU, UK but is now with LaTIM, INSERM, UMR 1101, Université de Bretagne Occidentale, Brest, France.
The aim of this work is to assess the feasibility of incorporating the Jacobian determinant in the similarity measure for accurate mass-preserving image registration, prior to using it in joint PET reconstruction and motion estimation.

\section{THEORY}

\section{A. Activity and density distributions}

Assume the tracer activity distribution $\lambda \in \mathcal{C}^{0}\left(\mathbb{R}^{3}, \mathbb{R}^{+}\right)$and density distribution $\mu \in \mathcal{C}^{0}\left(\mathbb{R}^{3}, \mathbb{R}^{+}\right)$. We define the standard warping operator $\mathcal{W}_{\varphi}: \mathcal{C}^{0}\left(\mathbb{R}^{3}, \mathbb{R}^{+}\right) \rightarrow \mathcal{C}^{0}\left(\mathbb{R}^{3}, \mathbb{R}^{+}\right)$associated to a diffeomorphism $\varphi: \mathbb{R}^{3} \rightarrow \mathbb{R}^{3}$ such that:

$$
\mathcal{W}_{\varphi}: f \mapsto f \circ \varphi \quad \text { where } f=\lambda \text { or } h=\mu .
$$

A mass-preserving warping operator $\widetilde{\mathcal{W}}_{\varphi}: \mathcal{C}^{0}\left(\mathbb{R}^{3}, \mathbb{R}^{+}\right) \rightarrow$ $\mathcal{C}^{0}\left(\mathbb{R}^{3}, \mathbb{R}^{+}\right)$can also be defined as:

$$
\widetilde{\mathcal{W}}_{\varphi}: h \mapsto\left|\operatorname{det}\left(\mathcal{J}_{\varphi}\right)\right| \cdot h \circ \varphi .
$$

where $\operatorname{det}\left(\mathcal{J}_{\varphi}(\boldsymbol{r})\right)$ is the determinant of the Jacobian matrix of the partial derivatives of $\varphi$ at a point $r \in \mathbb{R}^{3}$. The definition of this operator is motivated by the consideration of local mass preservation: if we consider a diffeomorphic deformation $\varphi$ which transforms a non-negative continuous distribution $f_{1}$ into $f_{2}$ (i.e., $f_{2}=f_{1} \circ \varphi$ ), then:

$$
\int_{\mathbb{R}^{3}} f_{1}(\boldsymbol{r}) \mathrm{d} \boldsymbol{r}=\int_{\mathbb{R}^{3}}\left|\operatorname{det}\left(\mathcal{J}_{\varphi}(\boldsymbol{r})\right)\right| f_{2}(\boldsymbol{r}) \mathrm{d} \boldsymbol{r},
$$

where $\operatorname{det}\left(\mathcal{J}_{\varphi}(\boldsymbol{r})\right)$ consequently reflects the volume changes. In this work, a B-spline parametrisation of $\varphi$ is used. The discretisation is performed after warping of the continuous distributions.

\section{B. Mass-Preserving Registration}

The optimisation problem consists in finding a deformation $\hat{\varphi}$ such that the similarities between two images $f$ and $g$ are increased, e.g., via minimisation of a similarity measure $\mathcal{C}$ :

$$
\hat{\varphi} \in \arg \min _{\varphi}\left\{\mathcal{C}\left(\widetilde{\mathcal{W}}_{\varphi} f, g\right)+\beta \mathcal{R}(\varphi)\right\},
$$

where $\mathcal{R}$ is a roughness penalty and $\beta$ its associated weight to ensure the estimation of a realistic deformation field. In this work, $\mathcal{C}$ is the sum of squared differences (SSD), as more suitable for a preliminary study before using log-likelihood in joint PET reconstruction and motion estimation [7]. Incorporating the Jacobian determinant into the similarity measure can be however challenging for noisy data, such as PET data [5]- the noise difference between images could be partly compensated 


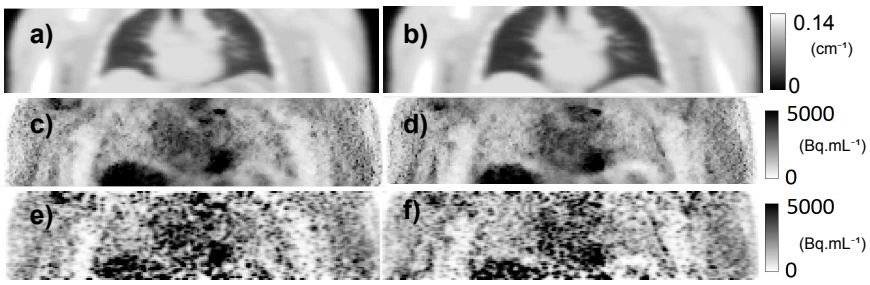

Fig. 1. Inputs: a) $\mu_{\exp }$, b) $\mu_{\text {insp }}$, c) end-expiration BSREM, d) end-inspiration BSREM, e) end-expiration OSEM, f) end-inspiration OSEM.

by changes in the Jacobian determinant. Two penalties on the Jacobian determinant image $\left|\operatorname{det} \mathcal{J}_{\varphi}\right|$ were considered:

- The quadratic Gibbs penalty [8]:

$$
\begin{aligned}
\mathcal{R}_{\mathrm{QP}}(\varphi) & \triangleq \\
& -\frac{1}{2} \sum_{j=1}^{n_{\mathrm{v}}} \sum_{k \in \mathcal{N}_{j}} \omega_{j, k}\left(\left|\operatorname{det} \mathcal{J}_{\varphi}\right|_{j}-\left|\operatorname{det} \mathcal{J}_{\varphi}\right|_{k}\right)^{2}
\end{aligned}
$$

where $n_{\mathrm{v}}$ is the voxel number, $\omega_{j, k}$ is the inverse distance between the centre of a voxel $j$ and the centre of a voxel $k$ and $\mathcal{N}_{j}$ is the neighbourhood of voxel $j$.

- The smoothed total variation (STV) penalty:

$$
\mathcal{R}_{\mathrm{STV}}(\varphi) \triangleq \sum_{j=1}^{n_{\mathrm{v}}} \sqrt{\left\|\nabla\left|\operatorname{det} \mathcal{J}_{\varphi}\right|_{j}\right\|_{2}^{2}+\zeta^{2}}, \quad \zeta>0
$$

where $\nabla\left|\operatorname{det} \mathcal{J}_{\boldsymbol{\alpha}}\right|_{j}$ is the (image) gradient of the absolute value of the Jacobian determinant at a voxel $j$ (the gradient approximation uses forward differences in $\mathcal{N}_{j}$ ) and $\zeta$ is used as a smoothing factor (here $\zeta=0.3$ ).

The gradients use the analytical derivatives of $\left|\operatorname{det}\left(\mathcal{J}_{\varphi}\right)\right|$, which were validated against finite differences. In both cases, a small quadratic penalty on the B-spline coefficients is added, to ensure that the deformation fields are globally smooth.

\section{EXPERIMENTS AND RESULTS}

\section{A. Patient Data}

FDG-PET listmode and CINE-CT data were acquired on a GE Discovery STE with monitoring using the Varian RPM system [2]. Both PET and CINE-CT data were gated into 5 bins based on the RPM displacement. The PET images were reconstructed with either (i) 60 iterations of OSEM, using 7 subsets and post-filtering or (ii) BSREM [9] (both including scatter and randoms modelling). The gated attenuation $(\mu)$ maps matched the PET gates. The input $\mu$, OSEM PET images and BSREM PET images used as input images are displayed in Figure 1.

In order to verify the stability of the registration, the PET data were divided into 4 parts (random gating), each part also gated into 5 respiratory bins. The end-expiration images were registered to the end-inspiration images using either quadratic regularisation or STV regularisation, for the three image types. Weak, average and strong regularisations (for both priors) were tested, normalised across PET and $\mu$ registrations using initial value of the cost function. All registration results presented incorporated mass preservation.
The registrations of the images corresponding to the entire acquisition were assessed by computing the normalised root mean squared deviation (NRMSD) between the warped endexpiration images and the end-inspiration images. Normalisations use the mean image value in the lung at end-inspiration.

The registrations of gated partial PET data were evaluated by plotting the variances in the Jacobian determinant images against the squared biases in the activity images. The variances in the activity image cannot be used as introducing the Jacobian determinant in the registration will tend to overfit the image noise, and therefore to lower variances (as discussed in [5]). Here $\boldsymbol{\lambda}_{\text {insp }}$ and $\boldsymbol{\lambda}_{\text {exp }}$ denote the end-inspiration and endexpiration block sequential regularized expectation maximization (BSREM) images, respectively. The measure of Jacobian image variances $\mathrm{Var}$ is given as

$$
\operatorname{Var}=\frac{1}{K-1} \frac{1}{N} \sum_{j=1}^{n_{\mathrm{v}}} \sum_{\kappa=1}^{K}\left(\left[\left|\operatorname{det} \mathcal{J}_{\varphi}\right|^{[\kappa]}\right]_{j}-m_{j}\right)^{2}
$$

where $K=16$ is the number of realisations and $\left|\operatorname{det} \mathcal{J}_{\varphi}\right|^{[\kappa]}$ corresponds to the Jacobian determinant image from the $\kappa$-th realisation and

$$
m_{j}=\frac{1}{K} \sum_{\kappa=1}^{K}\left[\left|\operatorname{det} \mathcal{J}_{\varphi}\right|^{[\kappa]}\right]_{j} .
$$

The (image) activity squared bias Bias ${ }^{2}$ is defined as

$$
\operatorname{Bias}^{2}=\frac{1}{K} \frac{1}{N} \sum_{j=1}^{n_{\mathrm{v}}}\left(\sum_{\kappa=1}^{K}\left[\dot{\mathcal{W}}_{\varphi} \boldsymbol{\lambda}_{\text {exp }}^{[\kappa]}\right]_{j}-\left[\boldsymbol{\lambda}_{\mathrm{insp}}\right]_{j}\right)^{2},
$$

where $\dot{\mathcal{W}}_{\varphi} \boldsymbol{\lambda}_{\exp }^{[\kappa]}$ corresponds to the end-expiration image $\boldsymbol{\lambda}_{\exp }^{[\kappa]}$ warped using the warping obtained from the $\kappa$-th registration and $\lambda_{\text {insp }}^{[\kappa]}$ is the input end-inspiration image from the $\kappa$-th realisation.

\section{B. Results}

In BSREM PET and $\mu$ registrations, weak and average regularisation for the two penalties showed similar results in terms of visual assessment of the alignment of the inner structures, however $\left|\operatorname{det}\left(\mathcal{J}_{\varphi}\right)\right|$ images appeared considerably smoother for the PET registration with average regularisation, and less prone to image noise. Strong regularisation failed to capture the motion appropriately and $\left|\operatorname{det}\left(\mathcal{J}_{\varphi}\right)\right|$ images were not realistic. OSEM PET registration showed poorer results, due to the lack of edge preservation and lower Signal to Noise Ratio than BSREM PET. The relative differences between mass-preserving and standard warping of $\mu_{\exp }$ and $\mu_{\mathrm{insp}}$ for STV regularisation are shown in Figure 2.

NRMSD measures in the lung-between the end-inspiration images and the end-expiration images warped using $\tilde{\mathcal{W}}-$ are given in Table I. $\left|\operatorname{det}\left(\mathcal{J}_{\varphi}\right)\right|$ images corresponding to an average penalisation (for STV prior) are shown in Figure 3.

We also compute the NRMSDs between the end-inspiration images and the end-expiration images warped using the standard operator $\mathcal{W}_{\varphi}$ instead of $\tilde{\mathcal{W}}_{\varphi}$ (where $\varphi$ is the deformation obtained from the mass-preserving registration). For an average STV regularisation, we have the NRMSD equal to $23.15 \%$ 


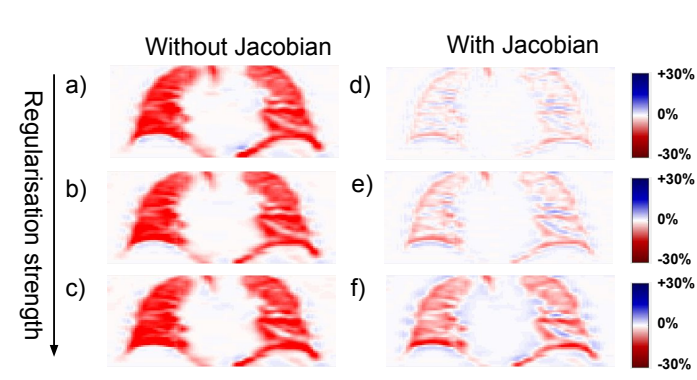

Fig. 2. Relative difference images between $\mu_{\exp } \circ \varphi$ and $\mu_{\text {insp }}$ (left) and between $\left.\mid \operatorname{det} \mathcal{J}_{\varphi}\right) \mid \cdot \mu_{\exp } \circ \varphi$ and $\mu_{\text {insp }}$ (right), for (a), (b) weak regularisation, (c), (d) average regularisation and (e), (f) strong regularisation using the STV penalty.

TABLE I

NRMSD BETWEEN THE TARGET IMAGES AND THE IMAGES WARPED USING THE MASS-PRESERVING OPERATOR FOR WEAK, AVERAGE AND STRONG REGULARISATIONS.

\begin{tabular}{|c|c|c|c|}
\hline STV & Weak & Average & Strong \\
\hline BSREM PET & $20.66 \%$ & $26.42 \%$ & $31.96 \%$ \\
\hline OSEM PET & $65.36 \%$ & $72.45 \%$ & $76.64 \%$ \\
\hline$\mu$ & $3.66 \%$ & $6.19 \%$ & $13.29 \%$ \\
\hline \hline Quadratic & Weak & Average & Strong \\
\hline BSREM PET & $20.74 \%$ & $26.46 \%$ & $32.01 \%$ \\
\hline OSEM PET & $65.42 \%$ & $72.48 \%$ & $76.64 \%$ \\
\hline$\mu$ & $3.60 \%$ & $6.26 \%$ & $13.47 \%$ \\
\hline
\end{tabular}

for $\mu$ and $31.19 \%$ for BSREM, up from $6.19 \%$ and $26.46 \%$, respectively.

The plots of the variance in the Jacobian maps against the bias in the activity images confirmed better results in the BSREM PET image registrations. As expected, using a stronger regularisation diminishes the variances but increases the biases (see figures 4 and 5 for BSREM). STV prior showed smaller variances in the entire image than the quadratic prior, although the variances in the lung were similar for both priors. The biases for the quadratic prior were slightly lower than for STV prior.

\section{Discussion ANd CONCLUSION}

Mass-preserving registration with a new regularisation scheme was evaluated on gated PET and CT images, for a standard "static" FDG scan of the thorax. Results indicate that the approximation of density and activity changes via

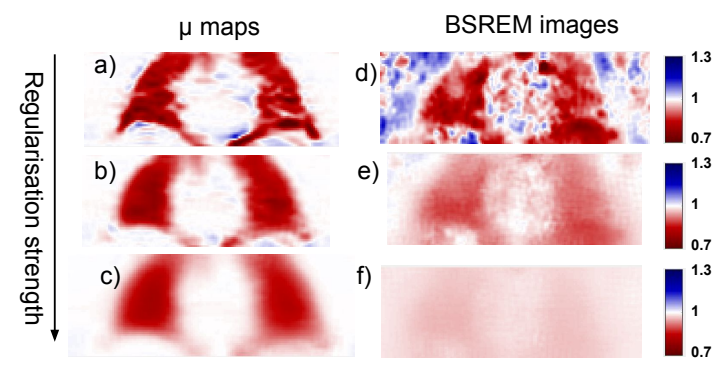

Fig. 3. Jacobian maps obtained with STV regularisation, for $\mu$ images: a) weak, b) average, c) strong; for BSREM PET images: d) weak, e) average, f) strong. The PET regularisation uses the activity images from the gated entire PET acquisitions.

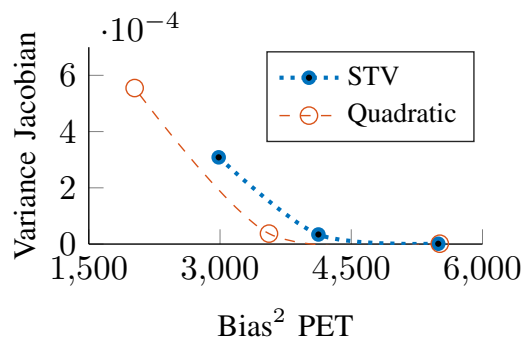

Fig. 4. Tradeoff between variance in the Jacobian determinant images and the (squared) bias in the activity images (in $\mathrm{Bq}^{2} / \mathrm{mL}^{2}$ ), in the entire image, for the BSREM sampled PET data.

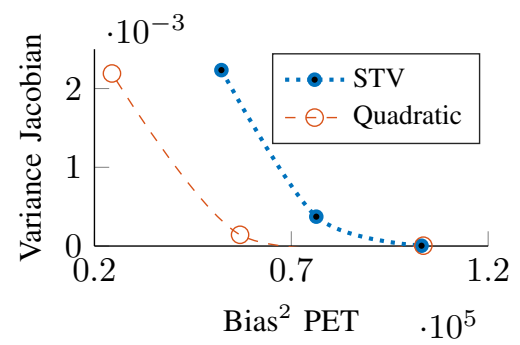

Fig. 5. Tradeoff between variance in the Jacobian determinant images and the (squared) bias in the activity images (in $\mathrm{Bq}^{2} / \mathrm{mL}^{2}$ ), in the lung only, for the BSREM sampled PET data.

volume changes (Jacobian determinant) provides good quantitative image values, especially in the lung, provided that regularisation is adequate. Using an edge-preserving image reconstruction helped with the registration and could be further optimised. Overall, both quadratic and STV priors performed well, although the STV prior showed reduced variances in the entire Jacobian maps, while the biases were reduced using the quadratic prior. The results here agree with [5]: (i) regularising is important for optimal registration and estimation of the Jacobian, otherwise volume changes could be underestimated [4], (ii) registration is more stable for less noisy input images, such as CT images or penalised PET images, where less regularisation is required. Future work will assess mass preservation in joint reconstruction.

\section{REFERENCES}

[1] B. A. Simon, "Non-invasive imaging of regional lung function using x-ray computed tomography.," Journal of clinical monitoring and computing, vol. 16, pp. 433-42, 2000.

[2] V. Cuplov et al., "Issues in quantification of registered respiratory gated PET/CT in the lung," Physics in Medicine and Biology, vol. 63, no. 1, 2018.

[3] A. Pépin et al., "Management of respiratory motion in PET/computed tomography: The state of the art," Nuclear Medicine Communications, vol. 35, no. 2, pp. 113-122, 2014.

[4] J. M. Reinhardt et al., "Registration-based estimates of local lung tissue expansion compared to xenon CT measures of specific ventilation," Medical Image Analysis, vol. 12, no. 6, pp. 752-763, 2008.

[5] K. Thielemans et al., "Mass-preserving image registration using freeform deformation fields," IEEE Nuclear Science Symposium Conference Record, pp. 2490-2495, 2009.

[6] F. Gigengack et al., "Motion correction in dual gated cardiac PET using mass-preserving image registration," IEEE Transactions on Medical Imaging, vol. 31, no. 3, pp. 698-712, 2012.

[7] A. Bousse et al., "Maximum-likelihood joint image reconstruction/motion estimation in attenuation-corrected respiratory gated PET/CT using a single attenuation map," IEEE Transactions on Medical Imaging, vol. 35, no. 1, pp. 217-228, 2016. 
[8] E. Ü. Mumcuoğlu et al., "Bayesian reconstruction of PET images: methodology and performance analysis," Physics in Medicine and Biology, vol. 41, no. 1, pp. 1777-1807, 1996.

[9] S. Ahn et al., "Globally convergent image reconstruction for emission tomography using relaxed ordered subsets algorithms," IEEE Transactions on Medical Imaging, vol. 22, no. 5, pp. 613-626, 2003. 